\title{
Predicting high risk of exacerbations in bronchiectasis: the E-FACED score
}

\author{
This article was published in the following Dove Press journal: \\ International Journal of COPD \\ 18 January 2017 \\ Number of times this article has been viewed
}

\author{
Martinez-Garcia MA, ${ }^{1,2}$ \\ Athanazio RA, ${ }^{3}$ Girón R, ${ }^{4}$ \\ Máiz-Carro $L,{ }^{5}$ de la Rosa $D,{ }^{6}$ \\ Olveira $C,{ }^{7}$ de Gracia J, ${ }^{2,8}$ \\ Vendrell M, ${ }^{9}$ Prados- \\ Sánchez $C,{ }^{10}$ \\ Gramblicka G," Corso \\ Pereira M, ${ }^{12}$ Lundgren FL, ${ }^{13}$ \\ Fernandes De Figueiredo $M,{ }^{14}$ \\ Arancibia $\mathrm{F}^{15}$ Rached $\mathrm{SZ}^{3}$
}

'Pulmonary Service, Polytechnic and University La Fe Hospital, Valencia, Spain; ${ }^{2}$ CIBERes, CIBER de Enfermedades Respiratorias. Madrid. Spain; ${ }^{3}$ Pulmonary Division, Heart Institute (Incor), Hospital das Clínicas da Faculdade de Medicina da Universidade de São Paulo; ${ }^{4}$ Pneumology Service, Hospital La Princesa, ${ }^{5}$ Pneumology Service, Hospital Ramón y Cajal, Madrid, 'Pneumology Unit, Hospital Plató, Barcelona, ${ }^{7}$ Pneumology, Málaga Regional University Hospital, Instituto de Biomedicina de Málaga (IBIMA), Málaga University, Spain; ${ }^{8}$ Pneumology Service, Hospital Vall d'Hebron, Barcelona, ${ }^{9}$ Bronchiectasis Group IDIBGI, Dr. Trueta University Hospital. UdG. Ciberes CB06/06/0030, ${ }^{10}$ Unidad de Fibrosis Quística y Bronquiectasias. Hospital Universitario La Paz. Madrid. Spain; ' Pneumology Service, Hospital del Tórax Dr A Cetrángolo, Buenos Aires, Argentina; ${ }^{12}$ Pneumology Service, Universidade Estadual de Campinas UNICAMP, Sao Paulo, ${ }^{13}$ Pneumology Service, Hospital Octávio de Freitas, Recife, ${ }^{14}$ Pneumology Service, Hospital de Messejana, Fortaleza, Brazil; ${ }^{15}$ Pneumology Service, Instituto Nacional del Tórax, Santiago de Chile, Chile

Correspondence: Martinez-Garcia MA Department of Pneumology, Hospital Universitario y Politécnico La Fe, Bulevar Sur s/n, 460I 2-Valencia, Spain

Tel +34 609865934

Email mianmartinezgarcia@gmail.com
Background: Although the FACED score has demonstrated a great prognostic capacity in bronchiectasis, it does not include the number or severity of exacerbations as a separate variable, which is important in the natural history of these patients.

Objective: Construction and external validation of a new index, the E-FACED, to evaluate the predictive capacity of exacerbations and mortality.

Methods: The new score was constructed on the basis of the complete cohort for the construction of the original FACED score, while the external validation was undertaken with six cohorts from three countries (Brazil, Argentina, and Chile). The main outcome was the number of annual exacerbations/hospitalizations, with all-cause and respiratory-related deaths as the secondary outcomes. A statistical evaluation comprised the relative weight and ideal cut-off point for the number or severity of the exacerbations and was incorporated into the FACED score (E-FACED). The results obtained after the application of FACED and E-FACED were compared in both the cohorts.

Results: A total of 1,470 patients with bronchiectasis ( 819 from the construction cohorts and 651 from the external validation cohorts) were followed up for 5 years after diagnosis. The best cut-off point was at least two exacerbations in the previous year (two additional points), meaning that the E-FACED has nine points of growing severity. E-FACED presented an excellent prognostic capacity for exacerbations (areas under the receiver operating characteristic curve: 0.82 for at least two exacerbations in 1 year and 0.87 for at least one hospitalization in 1 year) that was statistically better than that of the FACED score ( 0.72 and $0.78, P<0.05$, respectively). The predictive capacities for all-cause and respiratory mortality were 0.87 and 0.86 , respectively, with both being similar to those of the FACED.

Conclusion: E-FACED score significantly increases the FACED capacity to predict future yearly exacerbations while maintaining the score's simplicity and prognostic capacity for death.

Keywords: FACED score, E-FACED score, mortality, bronchiectasis, exacerbations

\section{Introduction}

Bronchiectasis is a multidimensional disease, and hence, its severity or prognosis could not be defined with any precision using a single variable. ${ }^{1-3}$ Accordingly, some recently published multidimensional scores combine clinical, functional, microbiological, and radiological variables to evaluate the prognosis and severity of bronchiectasis more comprehensively. ${ }^{2,3}$ One of these scores is the FACED score, ${ }^{3}$ an acronym derived from five dichotomized variables ( $\mathrm{F}$ : forced expiratory volume in $1 \mathrm{~s}\left[\mathrm{FEV}_{1}\right]$; A: age; C: chronic colonization by Pseudomonas aeruginosa [PA], E: radiological extension [number of pulmonary lobes affected], and D: dyspnea). The FACED score is easy to remember and to apply in clinical practice; it presents 
a range of values of growing severity spanning 0-7 points, and it has demonstrated an excellent prognostic capacity for mortality (both all-cause and respiratory) in both medium and long term, and an excellent capacity to discriminate different degrees of severity. ${ }^{4}$ It also had a good external validation in Europe as well as in various Latin American countries in bronchiectasis patients with wide-ranging etiology and severity and with characteristics different from those of the initial cohort. ${ }^{4-6}$ Nevertheless, some authors have found that the FACED score does not predict exacerbations accurately. ${ }^{6}$

One of the key clinical aspects in the prognosis and severity of airway diseases is exacerbations, particularly serious ones that require hospitalization. In bronchiectasis, exacerbations have been associated with higher mortality ${ }^{2}$ and a greater impairment of lung function, ${ }^{7}$ as well as more severe forms of the disease. ${ }^{8,9}$ Furthermore, another important aspect of exacerbations in these patients is the substantial concomitant health costs. ${ }^{10}$ As exacerbations are potentially preventable, exacerbations and hospitalizations are main outcomes in most of the clinical studies that seek to evaluate the efficacy and, above all, cost-efficiency of different treatments for bronchiectasis. ${ }^{1-16}$ Hence, tools that can predict future exacerbations and particularly those patients ar risk of presenting multiple exacerbations (especially severe ones requiring hospitalizations) are required.

Since the FACED score was not constructed with the variable "exacerbations," the present study aimed to analyze, in a wide-ranging series of patients different from the original FACED cohort, the prognostic capacity for exacerbations (and more particularly for patients with multiple exacerbations) of a new score called E-FACED and compare it with that of FACED. It also sought to evaluate whether E-FACED also maintains the FACED score's excellent predictive capacity for all-cause and respiratory mortality, as well as its capacity to discriminate different degrees of severity of bronchiectasis.

\section{Methods}

\section{Design}

This is an observational, multicenter study of two large historical cohorts involving seven teaching centers in Spain (construction series) and six teaching centers in Latin America (validation series), all with multidisciplinary and protocolized monographic bronchiectasis outpatient clinics.

\section{Patients}

The Spanish series corresponds with the one used for the construction and internal validation of the FACED score.
It comprised a total of 819 patients with bronchiectasis whose characteristics have previously been published. ${ }^{3}$ This series will be used as the construction cohort for the E-FACED. Meanwhile, 651 patients with bronchiectasis from six Latin American centers (four in Brazil, one in Argentina, and one in Chile) who were the subjects for the external validation of the FACED score will also be used for the external validation of the E-FACED score. The characteristics of these patients are listed in Table 1 . All the patients were diagnosed as having bronchiectasis by means of a primary diagnosis with HRCT, and they presented a wide range of severity, etiologies, and clinical and functional alterations, following the criteria described by Naidich et al. ${ }^{17}$ Patients aged $<18$ years were excluded, along with those whose vital state was unknown at the end of the follow-up. This study was approved by the Ethics and Research Committee of each participating center (Table S1).

Table I Comparative characteristics of the E-FACED score's construction and validation cohorts

\begin{tabular}{|c|c|c|}
\hline Variables & $\begin{array}{l}\text { Construction } \\
\text { cohort } \\
n=819(\%)\end{array}$ & $\begin{array}{l}\text { Validation } \\
\text { cohort } \\
n=651 \text { (\%) }\end{array}$ \\
\hline Age, years & $58.7(17.6)$ & $48.2(16)$ \\
\hline Gender, \% men & $356(43.5)$ & $214(32.9)$ \\
\hline Body mass index, $\mathrm{kg} / \mathrm{m}^{2}$ & $25.7(4.7)$ & $22.4(11.5)$ \\
\hline Dyspnea (mMRC) & $1.53(1.16)$ & $1.52(1.05)$ \\
\hline Smoking (pack-years) & $5.79(18.1)$ & $4.81(12.8)$ \\
\hline \multicolumn{3}{|l|}{ Appearance of sputum, $n(\%)$} \\
\hline Mucous & $199(24.5)$ & $177(27.2)$ \\
\hline Mucopurulent & $145(17.7)$ & $229(35.2)$ \\
\hline Purulent & $145(17.7)$ & $124(19)$ \\
\hline Respiratory failure, $\mathrm{n}(\%)$ & $83(10.1)$ & $110(16.9)$ \\
\hline Number of affected lobes & $2.52(1.2)$ & $3.37(1.5)$ \\
\hline Idiopathic bronchiectasis, n (\%) & $310(37.9)$ & $169(26)$ \\
\hline $\mathrm{FEV}_{1}, \%$ predicted & $68.9(25.9)$ & $54.7(22.1)$ \\
\hline FVC, \% predicted & $76.4(20.3)$ & $67.2(20.3)$ \\
\hline \multicolumn{3}{|l|}{ Chronic colonization, $\mathrm{n}(\%)$} \\
\hline Pseudomonas aeruginosa & $260(31.8)$ & $259(39.8)$ \\
\hline Haemophilus influenzae & $126(15.4)$ & $67(10.3)$ \\
\hline Multiresistant microorganism & $40(4.9)$ & $40(6.14)$ \\
\hline Isolation of Staphylococcus aureus, n (\%) & $42(5.1)$ & $49(7.5)$ \\
\hline Isolation of fungi, n (\%) & $178(21.7)$ & $39(6)$ \\
\hline Isolation of NTM, n (\%) & $23(2.8)$ & $8(1.3)$ \\
\hline Exacerbations (previous year)* & $2.52(2.2)$ & $1.12(1.4)$ \\
\hline Hospitalizations (previous year) & $0.7(1.2)$ & $0.4(0.8)$ \\
\hline Exacerbations/year (follow-up)* & - & $0.95(0.9)$ \\
\hline Hospitalization/year (follow-up) & - & $0.3(0.5)$ \\
\hline \multicolumn{3}{|l|}{ Chronic treatment, n (\%) } \\
\hline Systemic antibiotics & $59(7.2)$ & $46(7.2)$ \\
\hline Inhaled antibiotics & $146(17.8)$ & $198(30.5)$ \\
\hline Macrolides & $110(13.4)$ & $113(17.3)$ \\
\hline Oral corticoids & $39(4.7)$ & $25(3.8)$ \\
\hline Death, n (\%) & $154(18.8)$ & $95(14.6)$ \\
\hline
\end{tabular}

Note: *The number of hospitalizations (severe exacerbations) are not included. Abbreviations: mMRC, Modified Medical Research Council; NTM, non-tuberculous mycobacterium; $\mathrm{FEV}_{1}$, forced expiratory volume in I s; FVC, forced vital capacity. 


\section{Variables}

The variables recorded in both the construction cohort $(n=819)$ and the validation cohort $(n=651)$ were the same as those used in the original studies for the construction, internal validation, and external validation of the FACED score. In other words, the recorded variables corresponded as closely as possible to a radiological diagnosis of bronchiectasis. The exacerbations, hospitalizations, and microbiological data of the construction cohort pertained to the year prior to the patients' inclusion in the study, while these data were also available longitudinally for the validation cohort during the 5 years of the follow-up. Since there is no standard definition of an exacerbation, this study defined an exacerbation as a worsening of the respiratory symptoms concomitant with an increase in the volume or purulence of sputum that requires antibiotic treatment. This enabled identification of the number of exacerbations more objectively, according to the number of antibiotic treatments prescribed for changes in the volume or purulence of sputum or symptoms. Similarly, a severe exacerbation was defined as one that was considered by the physician to require hospitalization.

\section{Follow-up and main endpoints}

Patients from both cohorts were followed up for 5 years after the radiological diagnosis of bronchiectasis. After these 5 years, their vital state was determined. The number of deaths and their causes were identified via the hospitals' computerized records or the corresponding death certificates. The main variable was the prognostic capacity for exacerbations in a year, according to different cut-off points as regards number and severity. The secondary outcomes were established as the predictive capacity for death from any cause or from respiratory causes, and the capacity to discriminate different degrees of severity.

\section{Statistical analysis}

The SPSS 20.0 (IBM Corporation, Armonk, NY, USA) statistical package was used for all the calculations. The quantitative variables were evaluated as mean (standard deviation) in cases of normal distribution or as median (interquartile range) in other cases. The qualitative or dichotomic variables were evaluated as an absolute value (percentage of the total). The normality of the variables was determined by means of the Kolmogorov-Smirnov test. Various logistic regressions were made to determine the optimal cut-off point for the number of exacerbations and hospitalizations that would add the best independent prognostic value to the FACED score. The independent values in the construction series $(n=819)$, apart from the value of the FACED itself, the cut-off points of "at least one", "at least two", or "more than two" exacerbations or hospitalizations in the year prior to inclusion in the study, including a composite of "at least two exacerbations or one hospitalization" in the previous year. The dependent variable was the number of deaths from any cause in the Spanish construction cohort (819 patients). The prognostic capacity of the best score for the number or severity of the exacerbations was quantified by means of the odds ratio (OR; $95 \%$ confidence interval [95\% CI]), while the value of the OR, rounded up to its highest whole value, was used as a relative weight for the variable in the new score that was formed (E-FACED). In order to simplify the score, only different cut-off points were used for the number or severity of exacerbations, to make the variable dichotomic, as in the original FACED score.

Once the range of the E-FACED score had been calculated and obtained, its prognostic capacity for future exacerbations and hospitalizations over the course of a year (according to different cut-off points for number and severity) was determined by using the external validation database $(n=651)$ and calculating the corresponding areas under the receiver operating characteristic curve (AUCROC) and 95\% CI. A prognostic capacity was considered excellent if the AUC-ROC is $>80 \%$ of the total area. ${ }^{18}$ The prognostic capacity of the E-FACED for all-cause and respiratory mortality was then calculated, again by using the AUC-ROC. All the results were compared with those obtained from the application of the FACED score to the same series of patients. The ROC curves obtained from the E-FACED and FACED scores were compared by means of C-statistics. Finally, the Kaplan-Meier curves and the logrank test for comparing the curves two by two were used to determine the capacity of the new index (E-FACED) to discriminate the degree of severity, in order to divide bronchiectasis into mild (first tertile), moderate (second tertile), or severe (third tertile). In all cases, $P<0.05$ was considered significant.

\section{Results}

The construction cohort comprised 819 patients with bronchiectasis from the initial Spanish cohort for the construction and internal validation of the FACED, whereas the external validation cohort $(\mathrm{n}=651)$ comprised the Latin American cohort for the external validation of the FACED. Data from 20 patients from the initial Spanish cohort (2.4\%) and 21 patients from the Latin American cohort (3.1\%) were not obtained, mainly because of lack of information on their vital status at the end of the study (Figure 1). The differential characteristics of both cohorts are shown in Table 1. 


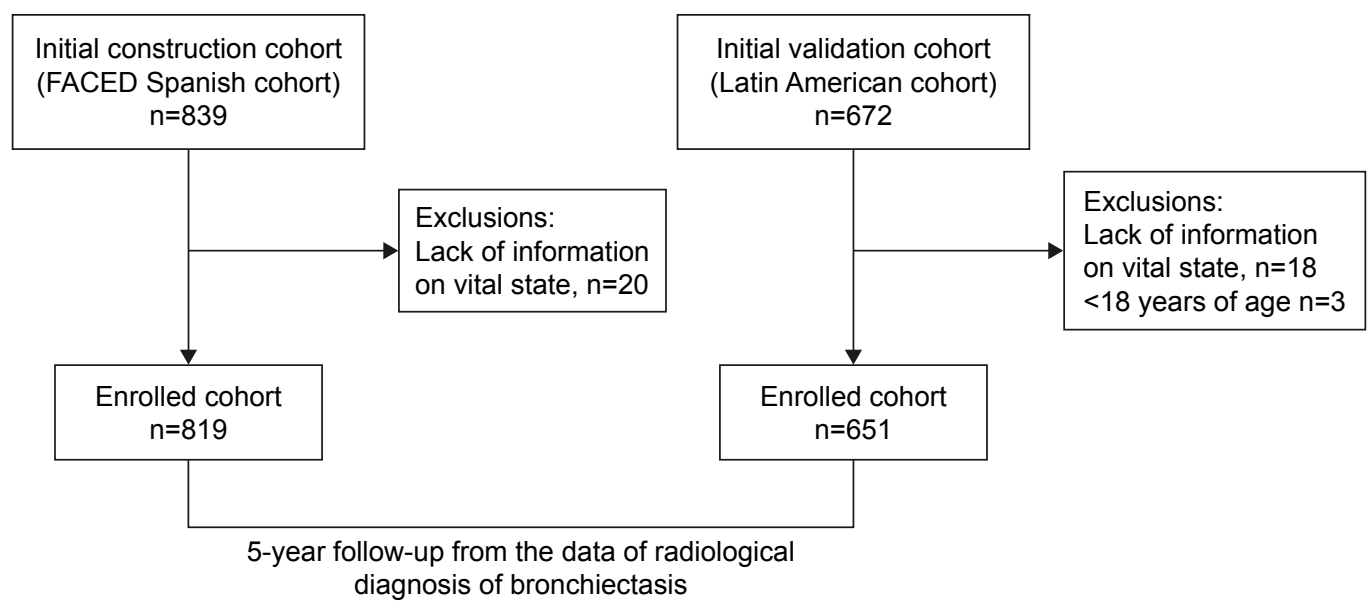

Figure I Flowchart of the study.

As can be seen, patients in the external validation cohort are younger, and they present greater clinical and functional severity, a greater radiological extension of bronchiectasis, a lower percentage of etiologies of unknown origin, and a significantly lower number of exacerbations.

\section{Construction of the E-FACED score}

Table 2 shows the results of the logistical regressions, which included different cut-off points for the number of exacerbations and hospitalizations in the previous year (at least one, at least two, or more than two, as well as a composite of two exacerbations or at least one hospitalization) to evaluate the FACED score's independent prognostic capacity for all-cause mortality. As can be seen, the cut-off point that added the most statistically significant prognostic value to the FACED score was "at least one hospitalization in the previous year," with an OR of 1.8, and so this was the variable chosen to be added to the FACED. This process therefore added two extra points to the FACED score in cases of at least one hospitalization in the previous year, or none in cases of no hospitalizations. Table 3 shows that the E-FACED score has a range of values of $0-9$ points. The three tertiles running from least to greatest severity are therefore $0-3$ points, 4-6 points, and 7-9 points.

Table 2 Different models of logistic regressions to determine the cut-off point for the number and severity of the exacerbations that adds the greatest prognostic capacity to the FACED score in the construction cohort $(n=819)$

\begin{tabular}{llll}
\hline & Model I & Model 2 & Model 3 \\
\hline FACED & $2.13(1.87-2.4 I)$ & $2.14(I .89-2.43)$ & $2.15(I .89-2.43)$ \\
& $P<0.000 \mathrm{I}$ & $P<0.000 \mathrm{I}$ & $P<0.000 \mathrm{I}$ \\
At least one hospitalization in the previous year, & $1.77(\mathrm{I} .22-2.6 \mathrm{I})$ & & \\
$\mathrm{n}=305$ (37.2\%) & $P=0.03$ & &
\end{tabular}

At least two hospitalizations in the previous year, $\quad 1.56(0.94-2.58)$

$\mathrm{n}=127(15.5 \%)$

$P=0.085$

Three or more hospitalizations in the previous year, $\mathrm{n}=68(8.3 \%)$

$1.33(0.79-1.49)$

\begin{tabular}{|c|c|c|c|c|}
\hline \multirow{2}{*}{$1=68(8.3 \%)$} & \multirow[b]{2}{*}{ Model 4} & \multicolumn{3}{|c|}{$P=0.1 /$} \\
\hline & & Model 5 & Model 6 & Model 7 \\
\hline \multirow[t]{2}{*}{ FACED } & $2.45(2.07-2.88)$ & $2.99(2.35-3.82)$ & $2.99(2.3-3.8)$ & $2.3(1.97-2.67)$ \\
\hline & $P<0.0001$ & $P<0.000$ I & $P<0.0001$ & $P<0.000$ I \\
\hline At least one exacerbation in the previous year, & $1.95(0.85-4.4)$ & & & \\
\hline $\mathrm{n}=693$ (84.6\%) & $P=0.1 \mathrm{I}$ & & & \\
\hline
\end{tabular}

I.18 (0.55-2.53)

$\mathrm{n}=525(64.1 \%) \quad P=0.66$

Three or more exacerbations in the previous year, $\mathrm{n}=326$ (39.8\%)

I.I $5(0.57-2.3)$

At least two exacerbations or at least one $P=0.69$

hospitalization in the previous year, $\mathrm{n}=446(54.4 \%)$

$1.66(0.82-3.34)$ $P=0.15$ 
Table 3 The E-FACED score

\begin{tabular}{lll}
\hline Variable & Values & Points \\
\hline At least one severe exacerbation & No & 0 \\
in previous year & Yes & 2 \\
FEV $_{\text {(\% predicted) }}$ & At least $50 \%$ & 0 \\
& $<50 \%$ & 2 \\
Age & $<70$ years & 0 \\
& At least 70 years & 2 \\
Chronic colonization by & Yes & $\mathrm{I}$ \\
Pseudomonas aeruginosa & No & 0 \\
Extension ( ${ }^{\circ}$ of pulmonary & I-2 lobes & 0 \\
lobes affected) & $>2$ lobes & 1 \\
Dyspnea (mMRC) & $0-I I$ & 0 \\
& III-IV & I \\
& Range & $0-9$ points \\
\hline
\end{tabular}

Abbreviations: FEV , forced expiratory volume in I s; mMRC, Modified Medical Research Council.

\section{Prognostic capacity for exacerbations}

Table 4 shows how the prognostic capacity of the E-FACED score was significantly greater than that of the FACED, in both the number and severity (hospitalizations) of the future exacerbations over the course of a year. The greatest benefit from E-FACED was found in the identification of patients with future multiple exacerbations (at least two exacerbations per year [Figure 2], or at least two exacerbations or one hospitalization per year).

\section{All-cause mortality: comparison of E-FACED and FACED - construction and validation cohorts}

Figure 3 shows the AUC-ROC of both the E-FACED and FACED scores for all-cause mortality in the initial construction cohort $(\mathrm{n}=819)$. These are $0.87(0.83-0.91)$ and 0.87 (0.82-0.91), respectively, with no significant differences between them; they are always $>0.80$, which means that they both present an excellent prognostic capacity. Furthermore, in the case of prognostic capacity for respiratory deaths, the results were again similarly excellent: the E-FACED score presented an AUC-ROC of 0.86 (0.81-0.9), whereas the FACED score presented one of $0.82(0.78-0.87)$.
Similarly, when both the E-FACED and the FACED scores were applied to the series of 651 patients from the Latin American cohorts, the AUC-ROCs were 0.84 (0.80-0.88) and $0.81(0.77-0.86)$, respectively, which are as excellent as those of the construction cohort, with no statistically significant differences between the two. Furthermore, in the case of the prognostic capacity for respiratory mortality, the E-FACED presented an AUC-ROC of 0.87 (0.83-0.91) and the FACED one of $0.84(0.80-0.88)$ (Figure 4$)$.

\section{Capacity to discriminate different degrees of severity}

It was also demonstrated (Figure 5) that the three tertiles of both the FACED and the E-FACED scores accurately differentiated patients into three groups of increasing severity, with no significant differences between the classifications created by the FACED and E-FACED scores.

Similarly, the comparative division of this validation cohort into three tertiles in both the E-FACED and the FACED scores also presented an excellent discriminatory capacity in three groups of increasing severity, with no significant differences from the construction cohort (Figure 6).

\section{Discussion}

According to the results of this study of two large series of patients with bronchiectasis, the addition to the FACED score of a simple dichotomized variable relating to the presence of previous severe exacerbations (hospitalizations; E-FACED score) significantly increases the prognostic capacity for future exacerbations and hospitalizations over the course of 1 year, making it a valid score for detecting patients with more exacerbated bronchiectasis. Furthermore, the addition of this variable does not make the score any more complex but does maintain its excellent prognostic capacity for both all-cause and respiratory mortality, while also retaining the capacity of FACED score to discriminate different degrees of severity. By adding a variable that is potentially modifiable or preventable with treatment, the E-FACED would broaden

Table 4 Comparison between the prognostic capacity of E-FACED and FACED for the number and severity of exacerbations in the validation cohort $(n=65 I)$

\begin{tabular}{|c|c|c|c|}
\hline & FACED & E-FACED & P-value \\
\hline At least one exacerbation per year $(n=228 ; 35 \%)$ & $0.70(0.67-0.75)$ & $0.76(0.72-0.80)$ & $<0.05$ \\
\hline At least two exacerbation per year $(n=\mid 17 ; 17.9 \%)$ & $0.72(0.68-0.78)$ & $0.82(0.78-0.87)$ & $<0.05$ \\
\hline At least one hospitalization per year $(n=56 ; 8.6 \%)$ & $0.82(0.78-0.87)$ & $0.89(0.85-0.92)$ & $<0.05$ \\
\hline $\begin{array}{l}\text { At least two exacerbations per year or one } \\
\text { hospitalization per year }(n=150 ; 23 \%)\end{array}$ & $0.78(0.74-0.82)$ & $0.87(0.83-0.90)$ & $<0.05$ \\
\hline
\end{tabular}

Note: Data are presented as AUC-ROC ( $95 \%$ confidence interval). 


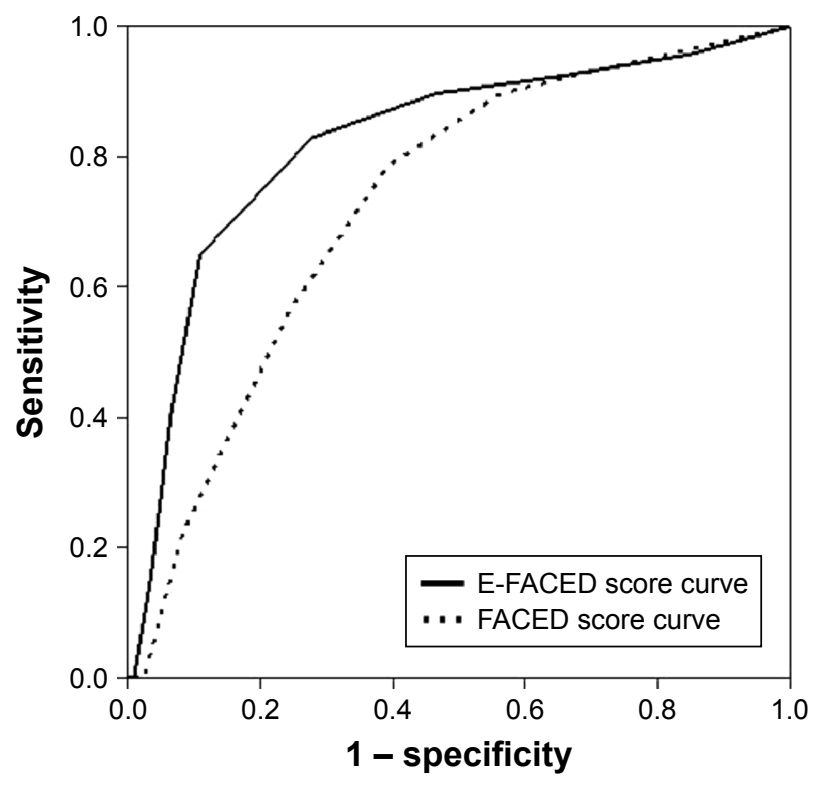

Figure 2 Comparative AUC-ROC for the prediction of at least two exacerbations per year.

Abbreviation: AUC-ROC, areas under the receiver operating characteristic curve.

its clinical applicability and its potential use in research studies on new treatments for bronchiectasis.

The FACED score is a very simple (and even memorizable) multilevel system for evaluating (without any need for a computer) the initial severity and prognosis of patients with bronchiectasis. ${ }^{3}$ However, one of its limitations is the fact that it does not cover exacerbations. And although there is little literature on this topic, some important studies have shown this variable to have a decisive impact, especially in its more severe forms, on both the severity and prognosis of bronchiectasis. ${ }^{2,6,7}$ In this respect, Chalmers et al, ${ }^{2}$ in a study of the construction and validation of the BSI (Bronchiectasis Severity Index) score, observed that both previous exacerbations (at least two exacerbations; HR (hazard ratio) 2.03 [1.02-4.03]) and, above all, severe exacerbations (hospitalization; HR 2.43 [1.02-4.03]) were significantly associated with an increase in all-cause mortality, with a very substantial relative weight (above all severe exacerbations) in the final value of the score. Although the BSI score is more complex than the FACED, it has demonstrated an excellent prognostic capacity for exacerbations/hospitalizations in patients with bronchiectasis. ${ }^{2}$ Moreover, Martínez-García et al have demonstrated that, over and above any chronic bronchial infection or presence of systemic inflammation, severe exacerbations were capable of producing a steep drop in lung function, of up to $120 \mathrm{~mL}$ of $\mathrm{FEV}_{1}$ in a year more than double the mean observed overall in patients with bronchiectasis ( $\sim 50 \mathrm{~mL} /$ year $).^{7}$

This substantial impact of exacerbations on bronchiectasis patients can be explained by the increase in diagnoses, ${ }^{19}$ related mortality, ${ }^{20}$ and hospitalizations due to bronchiectasis ${ }^{21,22}$ in recent years, even in industrialized countries, despite the excellent therapeutic arsenal at disposal. Ringshausen et $a 1,{ }^{21}$ for example, found a rise
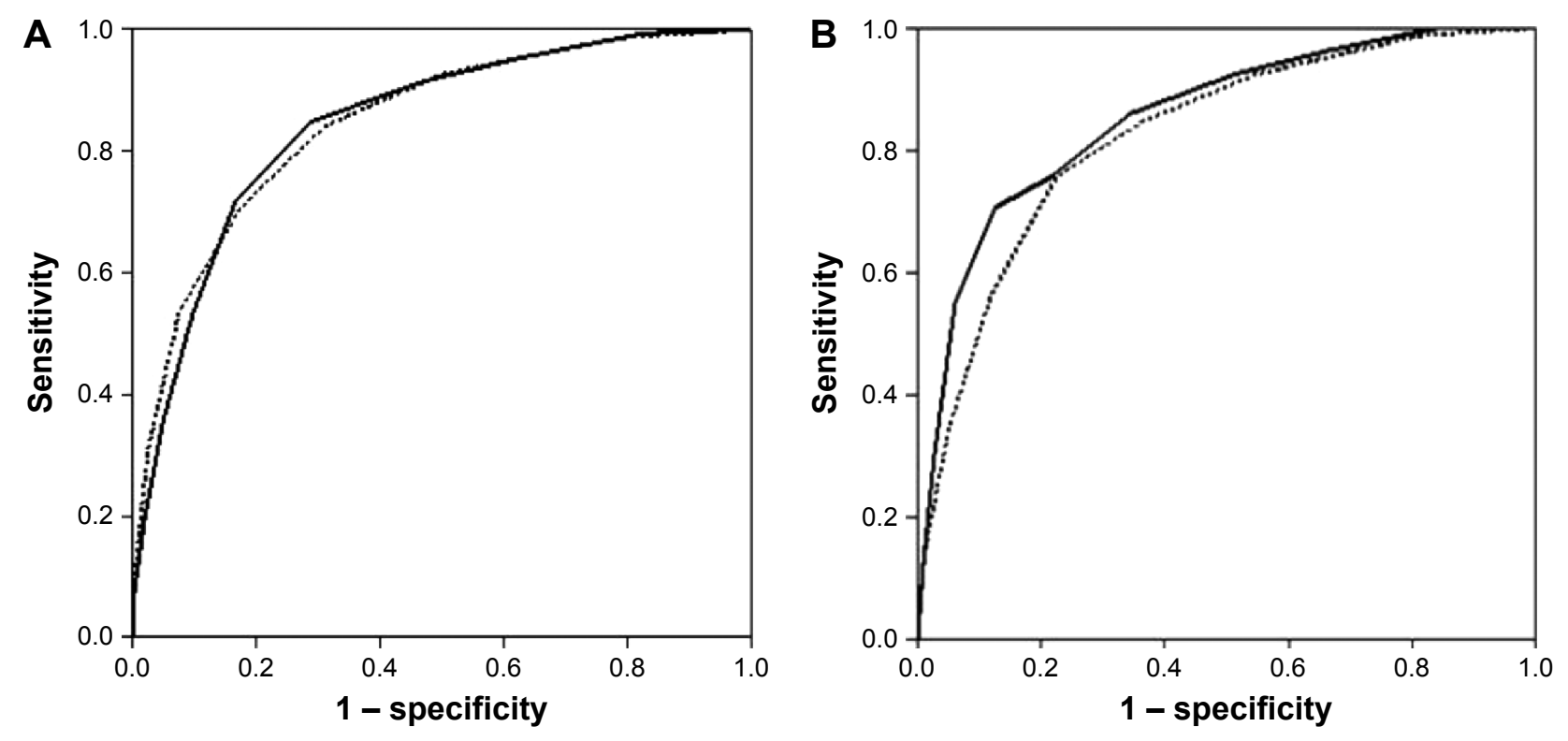

- E-FACED curve ..... FACED curve

Figure 3 Comparison of the prognostic capacity of the FACED and E-FACED scores for (A) all-cause mortality and (B) respiratory mortality in the initial cohort of patients $(n=819)$. 

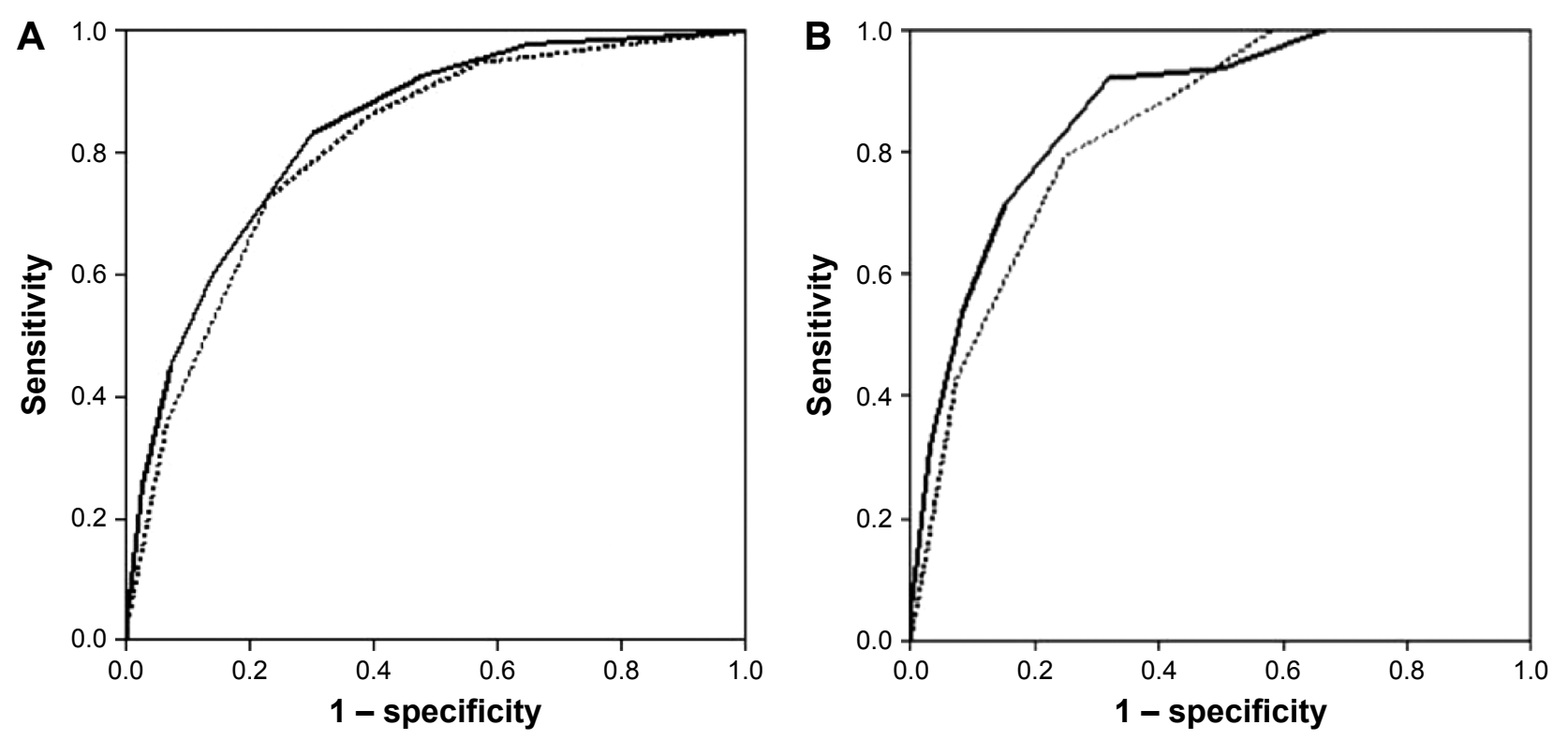

- E-FACED curve ..... FACED curve

Figure 4 Comparison of the prognostic capacity of the FACED and E-FACED scores for all-cause and respiratory mortality in the validation cohort ( $\mathrm{n}=65 \mathrm{I}$ ). Note: (A) All-cause mortality and (B) respiratory mortality.

in hospitalization from 9.4 to $39.4 / 100,000$ inhabitants, especially in women and the elderly. Similar findings have been observed in the USA, with an annual increase of $2.5 \%-3 \%$ in the period $1993-2006 .^{22}$

Furthermore, patients who present the greatest number or severity of exacerbations are also the ones who accumulate a higher percentage of health costs. De la Rosa et a ${ }^{10}$ found that the annual cost of treating a bronchiectasis patient in
Spain in 2012 was 4,671 euros, and 10,000 euros in cases of severe bronchiectasis (FACED 5-7 points), with exacerbations being one of the variables that explained a higher percentage of cost. Thus, the group of patients with more than two exacerbations $(21.5 \%)$ was responsible for $34.5 \%$ of the total cost of the 456 patients included, while those patients who required two or more hospitalizations $(5.7 \%)$ were responsible for $20.4 \%$ of the total cost. Therefore,
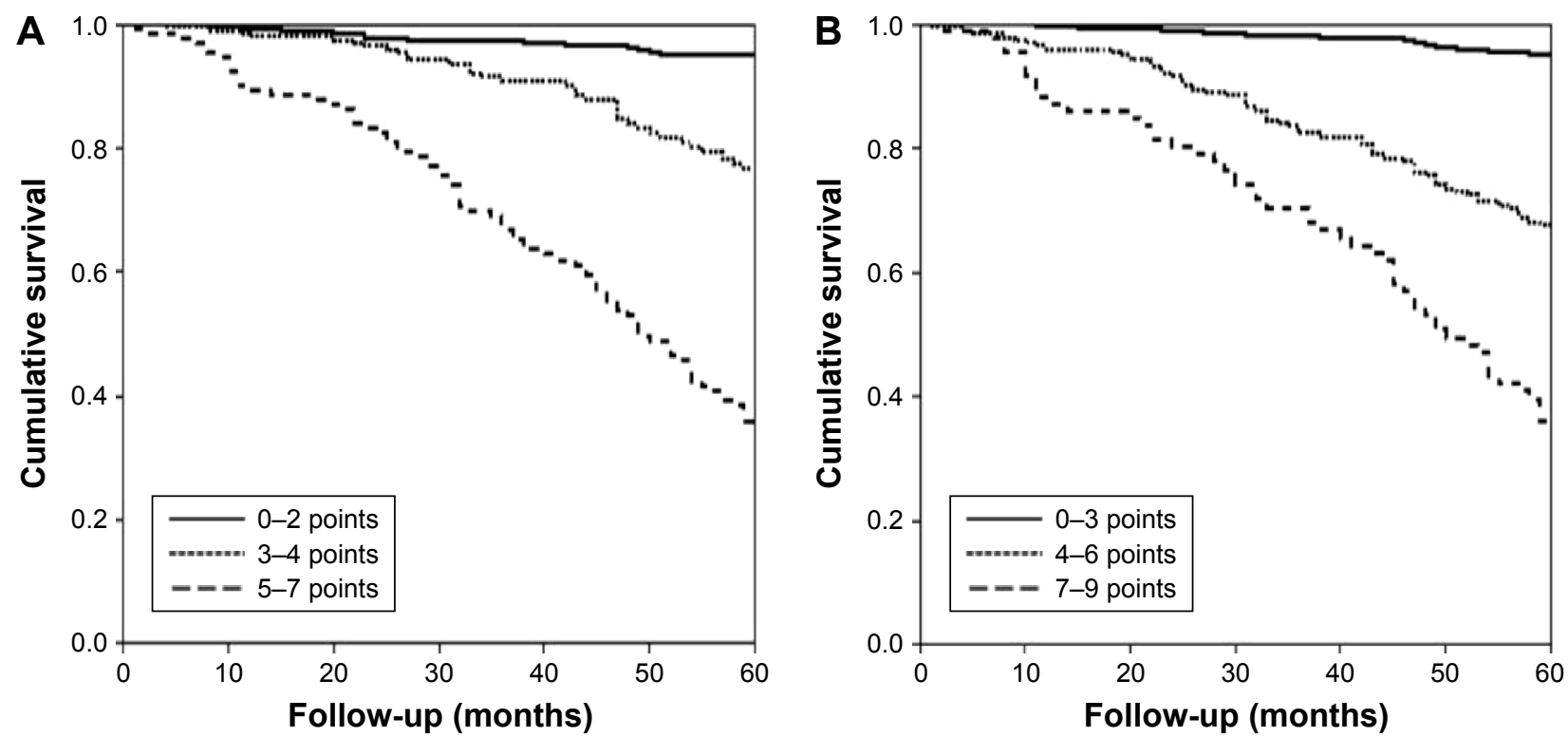

Figure 5 Comparison of the discriminatory capacity of the (A) FACED and (B) E-FACED scores in groups of increasing severity in the initial cohort ( $n=8$ I9).

Notes: (A) FACED score: mild: $0-2$ points, moderate: $3-4$ points, and severe: $5-7$ points. Log-rank test: mild bronchiectasis versus moderate bronchiectasis 5 I.2, $P<0.000$; mild versus severe 309.9; $P<0.000$ I; and moderate versus severe 64.6, $P<0.000$ I. (B) E-FACED score: mild: $0-3$ points, moderate 4-6 points, and severe 7-9 points. Log-rank test: mild bronchiectasis versus moderate bronchiectasis II I.2, $P<0.000$ I; mild versus severe 287.7; $P<0.000$ I; and moderate versus severe 26.5 , $P<0.000$ I. 

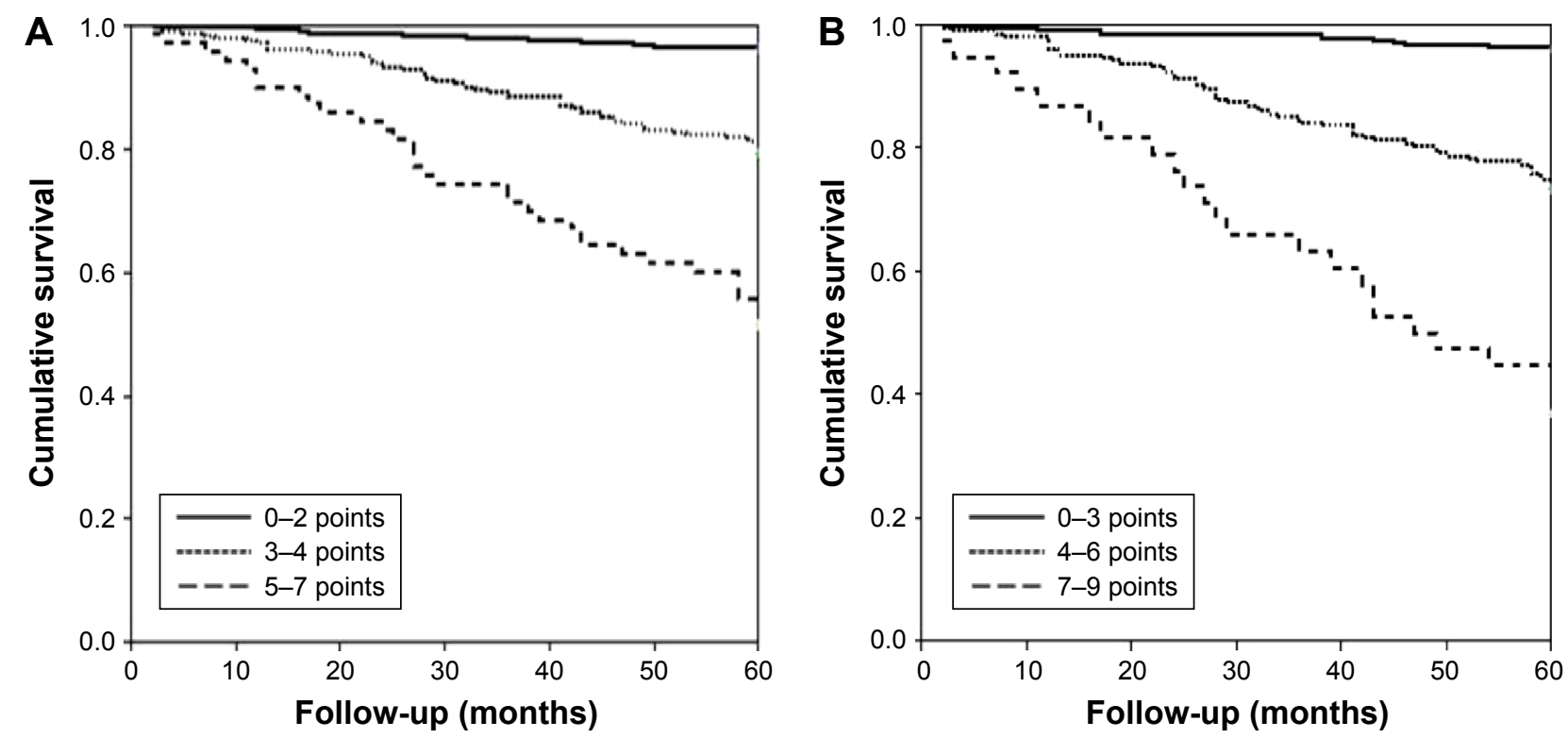

Figure 6 Comparison of the discriminatory capacities of the (A) FACED and (B) E-FACED scores in groups of increasing severity in the validation cohort ( $\mathrm{n}=65 \mathrm{I}$ ). Notes: (A) FACED score: mild: $0-2$ points, moderate: $3-4$ points, and severe: $5-7$ points. Log-rank test: mild bronchiectasis versus moderate bronchiectasis $43.3, P<0.000$; mild versus severe 138.9; $P<0.000$ I; and moderate versus severe 23.4, $P<0.000$ I. (B) E-FACED score: mild: $0-3$ points, moderate: $4-6$ points; and severe: $7-9$ points. Log-rank test: mild bronchiectasis versus moderate bronchiectasis $7 \mathrm{I} .3, P<0.000$ I; mild versus severe 201.2 ; $P<0.000$ I; and moderate versus severe 24 . I, $P<0.000$ I.

even without any definition of an exacerbatory clinical phenotype in bronchiectasis, the impact of exacerbations on these patients calls for a simple and prompt identification of the type of patients who will present a higher number or greater severity of exacerbations in the future.

In this study, the E-FACED score (a simple score that only adds to the FACED score one easily memorized dichotomic variable pertaining to the presence of hospitalizations in the year prior to the patient's diagnosis) presents advantages over the FACED. While maintaining the substantial prognostic capacity of the latter, for all-cause and respiratory mortality and for discrimination of degrees of severity, the E-FACED is capable, without accruing any greater complexity, of significantly improving the early identification of patients who will have at least two exacerbations or one hospitalization over the course of a year, or both, despite receiving treatment. This means that it is important to establish a prompt and thorough preventive strategy for these patients.

Moreover, another advantage of the E-FACED over the FACED is its greater susceptibility to change after the administration of different treatments. This may make it more suitable for research studies, both because it incorporates the main variable customarily used in randomized clinical trials (the number and severity of exacerbations) and because it presents a great prognostic capacity for these factors.

Although the present study has several significant strengths, particularly large numbers of patients being included in both the series (construction and external validation), it also presents a number of noteworthy limitations. On the one hand, exacerbations have been defined by the need for antibiotic treatment prescribed for changes in the volume or purulence of sputum or in the symptoms. They are therefore open to a more objective classification, although it should be emphasized that smaller exacerbations that do not require such treatment have also been recorded. It is nevertheless believed that the impact of this limitation on the conclusion cannot be very significant as most patients with exacerbations of bronchiectasis are treated with antibiotics, ${ }^{3}$ and furthermore, more severe exacerbations have the greatest impact on patients. ${ }^{2,6}$ On the other hand, the E-FACED was constructed using different cut-off points as regards the number of hospitalizations in the year prior to the patient's inclusion in the study, but no longitudinal data on the hospitalizations in the construction cohort $(n=819)$ in the subsequent years were available. Such longitudinal data on the number of yearly exacerbations and hospitalizations are available, however, for the validation cohort ( $n=651$ ), and, thanks to the way the E-FACED was constructed, it has demonstrated an excellent prognostic capacity. Moreover, we cannot exclude the influence of the chronic treatments used on clinical outcomes, especially in the number or severity of exacerbations. However, this is a limitation of all studies that include historical cohorts of patients with data recorded longitudinally from the time of diagnosis. Furthermore, no treatment has yet been proved to have any influence on the natural history of the disease. Finally, data were not obtained from $2.4 \%$ of 
patients from the original FACED score cohort and 3.1\% of patients from the Latin American series, mainly because of lack of information on their vital status, but such a small percentage of missing data cannot be expected to change the conclusion of the study.

\section{Conclusion}

In conclusion, the E-FACED offers a more versatile alternative to the FACED that is more susceptible to variations after medication, while maintaining the excellent prognostic capacity of the latter for mortality and its capacity to discriminate degrees of severity, without increasing the complexity of the score. Moreover, it presents a greater prognostic capacity for exacerbations and hospitalizations, especially in patients in which these are more frequent. Although further studies are required to investigate the applicability of the E-FACED in research studies, it is considered that the greater capacity of this score for change makes it more suitable than the FACED for use in clinical trials to evaluate different treatments for bronchiectasis.

\section{Author contributions}

Dr MA Martínez-García and Dr R Athanazio designed the study, contributed to data acquisition and interpretation, supervised the study, and wrote the manuscript. All authors contributed to data acquisition and interpretation, critically revised the manuscript, and approved the final version to be published.

\section{Disclosure}

The authors report no conflicts of interest in this work.

\section{References}

1. Martínez-García MA, Perpiñá-Tordera M, Soler-Cataluña JJ, RománSánchez P, Lloris-Bayo A, González-Molina A. Dissociation of lung function, dyspnea ratings and pulmonary extension in bronchiectasis. Resp Med. 2007;101:2248-2253.

2. Chalmers JD, Goemnne P, Aliberti S, et al. The Bronchiectasis Severity Index. An International derivation and validation study. Am J Respir Crit Care Med. 2014;189:576-585.

3. Martinez-Garcia MA, de Gracia J, Vendrell M, et al. Multidimensional approach to non-cystic fibrosis bronchiectasis: the FACED score. Eur Respir J. 2014;43:1357-1367.

4. Ellis HC, Cowman S, Fernandes M, Wilson R, Loebinger MR. Predicting mortality in bronchiectasis using bronchiectasis severity index and FACED scores: a 19-year cohort study. Eur Resp J. 2016;47: $482-489$.
5. Martinez-Garcia MA, Athanazio R, Gramblicka G, et al. International validation of FACED score in 672 patients with non-cystic fibrosis bronchiectasis. ATS Congress 2016, San Francisco. Abstract 10559.

6. McDonnell MJ, Aliberti S, Goeminne PC, et al. Multidimensional severity assessment in bronchiectasis: an analysis of seven European cohorts. Thorax. Epub 2016 Aug 11.

7. Martinez-Garcia MA, Soler-Cataluña JJ, Perpiná M, Román P, Soriano J. Factors associated with lung function decline in adult patients with stable non-cystic fibrosis bronchiectasis. Chest. 2007;132:1565-1572.

8. Martinez-Garcia MA, Vendrell M, Girón R, et al. The multiple faces of non-cystic fibrosis bronchiectasis: a cluster analysis approach. Ann Am Thorac Soc. 2016;13:1468-1475.

9. Aliberti S, Lonni S, Dore S, et al. Clinical phenotypes in adult patients with bronchiectasis. Eur Respir J. 2016;47:1113-1122.

10. De la Rosa D, Martinez-Garcia MA, Olveira C, Girón R, Maiz L, Prados C. Annual direct medical costs of bronchiectasis treatment: impact of severity, exacerbations, chronic bronchial infection and COPD coexistence. Chron Respir Dis. Epub 2016 Apr 12.

11. Altenburg J, de Graaff CS, Stienstra Y, et al. Effect of azithromycin maintenance treatment on infectious exacerbations among patients with non-cystic fibrosis bronchiectasis: the BAT randomized controlled trial. JAMA. 2013;309(12):1251-1259.

12. Barker AF, O'Donnell AE, Flume $\mathrm{P}$, et al. Aztreonam for inhalation solution in patients with non-cystic fibrosis bronchiectasis (AIR-BX1 and AIR-BX2): two randomised double-blind, placebo-controlled phase 3 trials. Lancet Respir Med. 2014;2(9):738-749.

13. Haworth CS, Foweraker JE, Wilkinson P, et al. Inhaled colistin in patients with bronchiectasis and chronic Pseudomonas aeruginosa infection. Am J Respir Crit Care Med. 2014;189(8):975-982.

14. Serisier DJ, Martin ML, McGuckin MA, et al. Effect of long-term, lowdose erythromycin on pulmonary exacerbations among patients with non-cystic fibrosis bronchiectasis: the BLESS randomized controlled trial. JAMA. 2013;309(12):1260-1267.

15. Wilson R, Welte T, Polverino E, et al. Ciprofloxacin dry powder for inhalation in non-cystic fibrosis bronchiectasis: a phase II randomised study. Eur Respir J. 2013;41(5):1107-1115.

16. Wong C, Jayaram L, Karalus N, et al. Azithromycin for prevention of exacerbations in non-cystic fibrosis bronchiectasis (EMBRACE): a randomised, double-blind, placebo-controlled trial. Lancet. 2012; 380(9842):660-667.

17. Naidich DP, McCauley DI, Khouri NF, Stitik FP, Siegelmen SS. Computed tomography of bronchiectasis. J Comput Assist Tomogr. 1982;6: $437-444$.

18. Hanley JA, McNeil BJ. The meaning and use of the area under a receiver operating characteristic (ROC) curve. Radiology. 1982;143:29-36.

19. Seitz AE, Olivier KN, Adjenian J, Holland SM, Prevots R. Trends in bronchiectasis among Medicare beneficiaries in the United States, 2000 to 2007 . Chest. 2012;142:432-439.

20. Roberts HJ, Hubbard R. Trends in bronchiectasis mortality in England and Wales. Resp Med. 2010;104:981-985.

21. Ringshausen FC, de Roux A, Pletz MW, Hämäläinen N, Welte T, Rademacher J. Bronchiectasis-associated hospitalizations in Germany, 2005-2011: a population-based study of disease burden and trends. PLoS One. 2013;8:e71109.

22. Seitz AE, Olivier KN, Steiner CA, Montes de Oca R, Holland SM, Prevots DR. Trends and burden of bronchiectasis-associated hospitalizations in the United States, 1993-2006. Chest. 2010;138:944-949. 


\section{Supplementary material}

Table SI List of ethical committees and approval numbers

\begin{tabular}{|c|c|c|}
\hline Center & Committee name & Approval number \\
\hline Heart Institute (Incor), Hospital das Clínicas da Faculdade & Cappesq & 1.267 .294 \\
\hline \multicolumn{3}{|l|}{ de Medicina da Universidade de São Paulo } \\
\hline \multirow[t]{3}{*}{ State University of Campinas (Unicamp), São Paulo, Brazil } & COMITÊ DE ÉTICA EM & I.142.117 \\
\hline & PESQUISA DA UNICAMP - & \\
\hline & CAMPUS CAMPINAS & \\
\hline Hospital del Tórax. Dr A Cetrángolo Buenos Aires, Argentina & Sala de Docencia e Investigaciôn & Vicente López, 20 de marzo de 2015 \\
\hline Hospital Octávio de Freitas Recife, Brazil & CAAE & 42747215.2 .1001 .5200 \\
\hline Hospital de Messejana Fortaleza, Brazil & CAAE & 42747215.2 .1001 .5200 \\
\hline Instituto Nacional del Tórax Santiago De Chile, Chile & CEC SSMO & 23082016 \\
\hline Spain FACED database. Main research Center: Hospital & Comité ético y de investigación & $0088-89-2011$ \\
\hline General Universitario (Valencia)* & & \\
\hline
\end{tabular}

Note: *This approval was obtained from the original database of FACED score, published in Eur Respir J. 20I4;43:1357.

\section{Publish your work in this journal}

The International Journal of COPD is an international, peer-reviewed journal of therapeutics and pharmacology focusing on concise rapid reporting of clinical studies and reviews in COPD. Special focus is given to the pathophysiological processes underlying the disease, intervention programs, patient focused education, and self management protocols.
Dovepress

This journal is indexed on PubMed Central, MedLine and CAS. The manuscript management system is completely online and includes a very quick and fair peer-review system, which is all easy to use. Visit $\mathrm{http}: / /$ www.dovepress.com/testimonials.php to read real quotes from published authors. 